\title{
A better definition of counselling strategies is needed to define effectiveness in temporomandibular disorders management
}

\author{
Abstracted from \\ de Freitas RF, Ferreira MA, Barbosa GA, Calderon PS. \\ Counselling and self-management therapies for temporomandibular disorders: a systematic review. \\ J Oral Rehabil 2013; 40: 864-874. Sep 18. doi: 10.1111/joor.12098. [Epub ahead of print] PubMed PMID: 24102692. \\ Address for correspondence: Rodrigo Falcão Carvalho Porto de Freitas, IFPB - Instituto Federal de Educação, \\ Ciência e Tecnologia da Paraíba - Campus Campina Grande, Av. Tranquilino C. Lemos, 671 - Dinamérica, \\ CEP 58432-300 - Campina Grande, PB - Brasil. E-mail: rodrigo_fcpf@hotmail.com
}

\section{Question: Is counselling and self-management effective in reducing the signs and symptoms of TMD?}

Data sources The Cochrane Library databases and Medline were searched along with the reference lists of identified articles and The Journal of the American Dental Association, Journal of Orofacial Pain and Journal of Oral Rehabilitation.

Study selection Controlled clinical trials in which the intervention included the treatment in at least one group with counselling techniques were included. Studies in English, Portuguese or Spanish were considered.

Data extraction and synthesis A standard data set was retrieved from the articles and a qualitative synthesis was undertaken.

Results Seven randomised controlled trials involving a total of 489 patients were included. Follow-up periods ranged from four weeks to 12 months. The studies suggested that counselling was able to improve tenderness upon masticatory muscle palpation and maximum mouth opening with and without pain in patients with TMD, with similar results to those of interocclusal appliances approaches.

Conclusions Although counselling- and self-management-based therapies could be a conservative low-cost and beneficial treatment alternative for treating TMD, with good results for the relief and control of TMD signs and symptoms by improving psychological domains and potentially reducing harmful behaviours, the evidence remains unclear due to the small number of controlled and randomised well-designed clinical trials. Thus, further studies with higher level of evidence and more representative samples should be conducted to validate the performance of this treatment modality.

\section{Commentary}

This systematic review summarised findings in the literature on the effectiveness of counselling and self-management strategies for temporomandibular disorders (TMD). The inclusion in the review was reserved to randomised controlled trials (RCTs) comparing those approaches with other treatment modalities. The authors included seven out of 581 potentially eligible papers identified in the PubMed and Cochrane databases, and concluded that counselling and self-management therapies may be a low-cost and beneficial treatment option in the field of TMD practice, also claiming the need for further studies on the topic. The search strategy and results were carefully reported. Neither a quality assessment nor a meta-analysis of findings of the included studies was performed due to the variability of methodological designs between the various investigations.

The efforts the authors made in reviewing the literature have been complicated by the objective difficulties of the argument. Notwithstanding that, some points can be raised to comment on the limitations of this review. Indeed, whilst the final message of the review is correct in claiming the potential effectiveness of counselling strategies as well as the need for further high-quality investigations, it seems that it derives more from the authors' a priori expectations than the reviewed evidence. The choice of including two of the seven papers was questionable, since they did not compare counselling with other treatments. In one paper, counselling was associated with all three tested regimens of occlusal appliance therapy, ${ }^{1}$ whilst in another paper the so-called 'usual treatment' also comprised medication with NSAIDs. ${ }^{2}$ Findings from the five remaining studies were also slightly different from that reported by the authors, and suggested that counselling/selfmanagement is better than (1 study) or equal to (1 study) oral appliance therapy, equal to physical therapy (1 study), moderately less effective than counselling plus physical therapy (1 study), and less effective than counselling plus posture training (1 study). The follow-up of the studies, and the selected populations were so different that it prevented any sound conclusions being achieved.

Apart from the considerations on the studies' selection and interpretation, the review's conclusions would have been much stronger if the authors had provided a clear definition of counselling, and if they had organised a valid framework to discuss their findings within the context of currently available concepts on TMD. In particular, the adoption of the unspecific term 'TMD' in most sections of the text and the lack of comments on the importance of the various clinical and psychosocial factors as predictors 
of treatment outcome, suggested that a better know-how of the overall TMD literature would be needed when performing reviews on selected topics. Examples of the suboptimal framework of the paper are numerous in the discussion, which contains mainly repetitions of the results and tables and does not consider the possibility that axis II diagnoses may influence the effectiveness of any counselling strategies. ${ }^{3,4}$

In conclusion, the authors should not have minded the risk of having only a few articles in their review, and should have provided examples of how to increase the quality of the available literature. To this purpose, creating hypotheses on which counselling/self-management strategies could be effective in which clinical conditions, may be useful suggestions for the design of future investigations.

Daniele Manfredini

Temporomandibular Disorders Clinic, Department of Maxillofacial Surgery, University of Padova, Italy
Practice points

- Counselling and self-management approaches may be effective strategies to manage TMD symptoms

- A better definition of the appropriate approach for each TMD condition is needed

- High quality literature on the topic is lacking.

1. Alencar F Jr, Becker A. Evaluation of different occlusal splints and counselling in the management of myofascial pain dysfunction. J Oral Rehabil 2009; 36: 79-85.

2. Truelove E, Huggins KH, Mancl L, Dworkin SF. The efficacy of traditional, low-cost and nonsplint therapies for temporomandibular disorder: a randomized controlled trial. I Am Dent Assoc 2006; 137: 1099-1107.

3. Manfredini D, Favero L, Del Giudice A, Masiero S, Stellini E, Guarda-Nardini L. Axis II psychosocial findings predict effectiveness of TMJ hyaluronic acid injections. Int J Ora Maxillofac Surg 2013; 42: 364-368.

4. Dworkin $\mathrm{SF}$, Huggins $\mathrm{KH}$, Wilson $\mathrm{L}$, et al. A randomized clinical trial using research diagnostic criteria for temporomandibular disorders-axis II to target clinic cases for a tailored self-care TMD treatment program. J Orofac Pain 2002; 16: 48-63.

Evidence-Based Dentistry (2013) 14, 118-119. doi:10.1038/sj.ebd.6400972 